Flores, P., Ambrósio, J., On the contact detection for contact-impact analysis in multibody systems. Multibody System Dynamics, Vol. 24(1), pp. 103-122, 2010. (DOI: $10.1007 / \mathrm{s} 11044-010-9209-8)$

\title{
On the contact detection for contact-impact analysis in multibody systems
}

\author{
PAULO FLORES ${ }^{1}$, JORGE AMBRÓSIO² \\ ${ }^{1}$ Departamento de Engenharia Mecânica, Universidade do Minho, \\ Campus de Azurém, 4800-058 Guimarães, Portugal \\ E-mail: pflores@dem.uminho.pt \\ ${ }^{2}$ Departamento de Engenharia Mecânica, Instituto Superior Técnico, \\ IST/IDMEC, Av. Rovisco Pais, 1, 1049-001 Lisboa, Portugal \\ E-mail: jorge@dem.ist.utl.pt
}

\begin{abstract}
One of the most important and complex parts of the simulation of multibody systems with contactimpact involves the detection of the precise instant of impact. In general, the periods of contact are very small and, therefore, the selection of the time step for the integration of the time derivatives of the state variables plays a crucial role in the dynamics of multibody systems. The conservative approach is to use very small time steps throughout the analysis. However, this solution is not efficient from the computational view point. When variable time step integration algorithms are used and the pre-impact dynamics does not involve high-frequencies the integration algorithms may use larger time steps and the contact between two surfaces may start with initial penetrations that are artificially high. This fact leads either to a stall of the integration algorithm or to contact forces that are physically impossible which, in turn, lead to post-impact dynamics that is unrelated to the physical problem. The main purpose of this work is to present a general and comprehensive approach to automatically adjust the time step, in variable time step integration algorithms, in the vicinity of contact of multibody systems. The proposed methodology ensures that for any impact in a multibody system the time step of the integration is such that any initial penetration is below any prescribed threshold. In the case of the start of contact, and after a time step is complete, the numerical error control of the selected integration algorithm is forced to handle the physical criteria to accept/reject time steps in equal terms with the numerical error control that it normally uses. The main features of this approach are the simplicity of its computational implementation, its good computational efficiency and its ability to deal with the transitions between non contact and contact situations in multibody dynamics. A demonstration case provides the results that support the discussion and show the validity of the proposed methodology.
\end{abstract} Keywords: Contact detection, Contact-Impact analysis, Time integrators, Integration Error Control, Variable time step, Multibody dynamics. 


\section{Introduction}

The classical problem of the contact mechanics is still an open issue in engineering applications. In particular, the contact-impact modeling and analysis in multibody dynamics has received a great deal of attention over the past few decades and still remains an active field of research and development [1-6]. Contact events happen frequently in multibody systems and in many cases the function of mechanical systems is based on them [7-15]. In general, the motion characteristics of a multibody system are significantly affected by contact-impact phenomena. Impact is a complex physical phenomenon for which the main characteristics are a very short duration, high force levels, rapid energy dissipation and large changes in the velocities of the bodies [16]. Inherently, contact implies a continuous process which takes place over a finite time. Other effects directly related to the impact phenomena are those of vibration propagation through the system, local elastic/plastic deformations at the contact zone and frictional energy dissipation [17-26]. Impact is a prominent phenomenon in many mechanical systems such as mechanisms with intermittent motion and mechanisms with clearance joints [27-31]. In a broad sense, the contact-impact modeling in multibody systems consists of two major steps, namely, the contact detection and contact response.

The subject of development of contact detection problem is a quite challenging and actual problem in various fields such as, discrete element methods [32], robotics [33] or vehicle systems [34]. From the modeling methodology point of view, several different methods have been developed. Carsten and Wriggers [35] presented an explicit multibody contact algorithm where the contact detection issue was also studied using a predictor-corrector scheme. An iterative form of the proposed scheme was also used to reduce the computational effort. One of the most robust and well known methods for contact detection of complexly shaped bodies was proposed by Hippmann [36]. This algorithm, referred to as polygonal contact model, is based on representation of the body surfaces by polygon meshes and the contact force evaluation is done using an elastic foundation model. This approach has been used by other researchers [37, 38]. He et al. [39] presented a multigrid contact detection method, where the multigrid idea was integrated with contact detection problems. Wellmann et al. [40] developed an efficient contact a contact detection algorithm for superellipsoids based on the common-normal concept. The problem of contact detection is formulated as 2D unconstrained optimization problem that is solved by a combination of Newton's method and a Levenberg-Marquardt method. More recently, Studer et al. [41] extended the modern time stepping algorithms to include a step size adjustment and extrapolation for contact detection in non-smooth dynamics. Portal et al. [42] presented a methodology for contact detection between convex quadric surfaces using its implicit equations. This methodology was implemented in a multibody dynamics code in order to simulate the interpenetration between mechanical systems, particularly, the simulation of collisions with automotive vehicles and other road users, such as cars, motorcycles and pedestrians. The contact 
detection of two bodies was formulated as a convex nonlinear constrained optimization problem that is solved using two methods, an Interior Point method (IP) and a Sequential Quadratic Programming method (SQP).

From the modeling methodology point of view, several different methods have been introduced to model the contact response in multibody systems. As a rough classification, they may be divided into contact force based methods [43] and methods based on geometrical constraints [44], each of them showing advantages and disadvantages for each particular application.

Contact force approaches, commonly referred as penalty or compliant methods, own their importance in the context of multibody systems with contacts to their computational simplicity and efficiency [17]. In these methods, the contact force is expressed as a continuous function of penetration between contacting bodies. One of the main drawbacks associated with these force models is the difficulty to choose contact parameters such as the equivalent stiffness or the degree of nonlinearity of the penetration, especially for complex contact scenarios and nonmetallic materials [45]. The penalty formulations can be understood as if each contact region of the contacting bodies is covered with some spring-damper elements scattered over its surfaces. The normal force, including elastic and damping, prevents penetration, i.e., no explicit kinematic constraint is considered but simply force reaction terms are used. The magnitudes of stiffness and deflection of the spring-damper elements are computed based on the penetration, material properties and surface geometries of the colliding bodies. In the work by Khulief and Shabana [28, 29] the required parameters for representing contact force laws are obtained based on the energy balance during contact. This formulation uses a force-displacement law that involves determination of material stiffness and damping coefficients. In the work by Lankarani and Nikravesh, [46] two continuous contact force models are presented for which unknown parameters are evaluated analytically. In the first model, internal damping of bodies represents the energy dissipation at low impact velocities. However, in the second model local plasticity of the surfaces in contact becomes the dominant source of energy dissipation. Dias and Pereira [47] described the contact law using a continuous force model based on the Hertz contact law with hysteresis damping. The effect and importance of structural damping schemes in flexible bodies were also considered. Hunt and Crossley [48] obtained a model for computing the stiffness coefficient from the energy balance relations. Based on the Hunt and Crossley approach Lankarani and Nikravesh [43] further extend the contact model with hysteresis damping. In their approach, the damping force is a linear function of the elastic penetration which is estimated from the energy dissipated during impact. The effect of friction in this approach is often taken into consideration by using a regularized Coulomb friction model. An overview of different models of friction together with fundamentals can be found in Oden and Martins [49] and Feeny et al. [50]. 
The complementarity formulations associated with the Moreau's time-stepping algorithm for contact modeling in multibody systems have used by many researchers $[3,10,32,38,41]$. Assuming that the contacting bodies are truly rigid, as opposed to locally deformable or penetrable bodies as in the penalty approaches, the complementarity formulations resolve the contact dynamics problem by using the unilateral constraints to compute contact impulses or forces to prevent penetration from occurring. Thus, at the core of the complementarity approach is an explicit formulation of the unilateral constraints between the contacting rigid bodies [51]. The basic idea of complementarity in unilateral multibody systems can be stated as for a unilateral contact either relative kinematics is zero and the corresponding constraint forces are zero, or vice versa. The product of these two groups of quantities is always zero. This leads to a complementarity problem and constitutes a rule which allows the treatment of MBS with unilateral constraints [52-55]. One of the first published works on the complementarity problems is due to Signorini [56], who introduced an impenetrability condition in the form of a Linear Complementary Problem. Later, Moreau [57] and Panagiotopoulos [58] also applied the concept of complementarity to study nonsmooth dynamic systems. Pfeiffer and Glocker [7] extended the developments of Moreau and Panagiotopoulos to multibody dynamics with unilateral contacts, being the complementarity considered of paramount importance. Indeed, complementarity problems proved to be a very useful way to formulating problems involving discontinuities [59-62].

In a dynamic simulation it is very important to find the precise instant of transition between the different states, that is, the transition between contact and non-contact situations. Especially when continuous contact force models are used, such as the one proposed by Lankarani and Nikravesh [43], if the instant of the start of contact is not detected properly the initial contact force may become abnormally large due to the unphysical high initial penetrations between the impacting surfaces. This numerical abnormality leads to an artificial increase of the system energy and, eventually, to the stall of the integration process, when variable time step integration algorithms are used. The avoidance of this problem requires a close monitoring of the numerical procedure to continuously detect and analyze all situations. Otherwise, the errors may buildup and the final results are meaningless. Thus, the main purpose of this work is to present a general and comprehensive methodology to deal with the detection of the precise instant of contact in multibody dynamics and to propose actions at the level of the integration algorithm that, without interfering with its mathematical structure, allows controlling time steps based on physical reasoning as a complement of the time-step control inherent to all variable time stepping integration algorithms. 


\section{Model for Contact Forces}

In order to evaluate efficiently the contact-impact forces resulting from collisions in multibody systems special attention must be given to the numerical description of the contact force model. Information on the impact velocity, material properties of the colliding bodies and geometry characteristics of the contact surfaces must be included into the contact force model. Due to its simplicity and ability to characterize the contact phenomena the contact forces are represented, in this work, using a continuous force model based on a penalty formulation [43]. Further, it is important that the contact force model can add to the stable integration of the multibody system equation of motion. This contact force model is based on the Hertz elastic contact law, being the hysteresis damping function incorporated to represent the energy dissipated during the impact. Lankarani and Nikravesh [43] suggest separating the normal contact force into elastic and dissipative components as,

$$
f_{N}=K \delta^{n}+D \&
$$

where $K$ is the generalized stiffness constant and $\delta$ is the relative normal indentation between the bodies. The exponent $n$ is set to 1.5 for the cases where there is a parabolic distribution of contact stresses, as in the original work by Hertz [63]. Convenient expressions for the contact force based on experimental or numerical work use $n=1.5$, for metallic materials, and other exponents for other materials such as glass or polymers. Although such penalty formulations have the same form of Equation (1) and they pose the same numerical challenges if the exponent $n \neq 1.5$ or the distribution of the contact stresses is not parabolic they must not be confused with the Hertz theory. The generalized parameter $K$ is dependent on the material properties and the shape of the contact surfaces. For two spheres in contact the generalized stiffness coefficient is function of the radii of the spheres $i$ and $j$ and the material properties as [64],

$$
K=\frac{4}{3\left(\sigma_{i}+\sigma_{j}\right)}\left[\frac{R_{i} R_{j}}{R_{i}+R_{j}}\right]^{\frac{1}{2}}
$$

where the material parameters $\sigma_{i}$ and $\sigma_{j}$ are given by,

$$
\sigma_{k}=\frac{1-v_{k}^{2}}{E_{k}}, \quad(k=i, j)
$$

and the quantities $v_{k}$ and $E_{k}$ are the Poisson's ratio and the Young's modulus associated with each sphere, respectively.

In Equation (1) the quantity $D$ is a hysteresis coefficient and $\dot{\delta}$ is the relative normal impact velocity. The hysteresis coefficient is written as a function of penetration as,

$$
D=\chi \delta^{n}
$$

in which the hysteresis factor $\chi$ is given by, 


$$
\chi=\frac{3 K\left(1-c_{e}^{2}\right)}{4 \xi^{(-)}}
$$

being $\dot{\delta}^{(-)}$the initial impact velocity. By substituting Equation (5) into Equation (4) and the result into Equation (1), the normal contact force is finally expressed as,

$$
f_{N}=K \delta^{n}\left[1+\frac{3\left(1-c_{e}^{2}\right)}{4} \frac{\delta^{\&}}{\delta^{\&-1}}\right]
$$

where the generalized parameter $K$ is evaluated by Equation (2) for sphere to sphere contact, or by similar expressions for the contact of other types of geometry and $c_{e}$ is the restitution coefficient. Therefore, it is crucial, for the correct use of the continuous force model, the exact identification of the initial contact velocity $\dot{\delta}^{(-)}$and to start the analysis of the contact period with a null penetration $\delta$, which in numerical terms means a penetration smaller than a pre-defined tolerance, i.e., $\delta<\varepsilon$.

\section{Contact Detection Methodology}

When a system consists of fast and slow moving components, that is, the eigenvalues are widely spread, the system is designated as being stiff [65]. Stiffness in the system equations of motion arises when the gross motion of the overall multibody system is combined with the nonlinear contact forces that lead to rapid changes in velocity and accelerations. In addition, when the equations of motion are described by a coupled set of differential and algebraic equations, the error of the response system is particularly sensitive to constraints violation, which inevitably leads to artificial and undesired changes in the energy of the system. Yet, by applying a stabilization technique the constraint violation can be kept under control [66]. During the numerical integration procedure, both the order and the step size of the integration algorithms are adjusted to keep the error tolerance under control. In particular the variable step size of the integration scheme is a desirable feature when integrating systems that exhibit different time scales, such as in multibody systems with impacting bodies [67]. Thus, large steps are generally taken when the motion of the system does not include contact forces but, when impact occurs, the step size is decreased substantially due to the inclusion of high frequency contents in the system response and not because the amount of penetration observed between two contact surfaces is larger or smaller.

One of the most critical aspects in the dynamic simulation of the multibody systems with collisions is the detection of the precise instant of contact. In addition, the numerical model used to characterize the contact between the bodies requires the knowledge of the pre impact conditions, that is, the impact velocity and the direction of the normal to the colliding surface. Neither the contact duration nor the penetration can be predicted from the pre-impact conditions due to the influence of the kinematic constraints and other interactions on the bodies of the complete system. Take, for instance, a system in which before the first impact, not only the bodies move slowly relative to each other but also 
the complete system is moving with an almost constant velocity. In this phase, the step size of the integration algorithm may become relatively large, being the global motion of the system characterized by relatively large translational and rotational displacements during a single time step. Therefore, if the numerical integration is not handled properly, the first impact between the colliding bodies may be observed with a high penetration depth, and, hence, the contact forces evaluated with penalty formulations become artificially large.

The importance of the initial penetration control, in the framework of the integration of the equations of motion, is better discussed using a simple example. Take the case of the falling ball illustrated in Figure 1, with a mass of $m=1.0 \mathrm{~kg}$, a moment of inertia equal to $0.1 \mathrm{~kg} \mathrm{~m}^{2}$, a radius $R=0.1 \mathrm{~m}$, animated by an initial horizontal velocity $v=1.0 \mathrm{~m} / \mathrm{s}$ and acted upon by gravity forces only. The motion of the ball is such that during its falling trajectory it strikes the ground. The penetration of the ball in the ground, in the integration time step, for which contact is first detected, is,

$$
\delta^{(-)}=y_{b}-R
$$

where $y_{b}$ is the $y$ coordinate of the ball center of mass. The superscript (-) on $\delta$ means that it is the penetration when contact is first detected. Note that $\delta^{(-)}$must has a positive value for contact. Therefore, by monitoring the sign of the penetration at every time step $t+\Delta t$ the start can be identified from,

$$
\delta^{(-)}(\mathbf{q}, t) \delta^{(-)}(\mathbf{q}, t+\Delta t) \leq 0
$$

When Equation (8) is satisfied the start of contact is defined as occurring at $t+\Delta t$. The integration of the equations of motion of the system may proceed with no numerical problem if the penetration first detected is close to zero, or at least below a pre-defined threshold, i.e., if $\delta^{(-)}(\mathbf{q}, t+\Delta t) \leq \delta_{\max }$. When the initial penetration detected is close to zero the trajectory of the ball, illustrated in Figure 1, is such that it rebounds to a height close or equal to that of its release. When the initial penetration happens to be large enough there is gain of energy leading the ball to bounce to an height higher than its release. These two situations are shown in Figure 1(b) and 1(c). Because it cannot be left to chance, strategies to limit the time step in the vicinity of contact must be implemented when solving contact problems. Certainly, the conservative approach of only using small time steps can solve many problems, but it defeats the original reasons to use variable time step integration algorithms in multibody dynamics. In this work a time-step control procedure based on physical reasoning that complements the numerical error time-step control of the integration algorithm is proposed. 


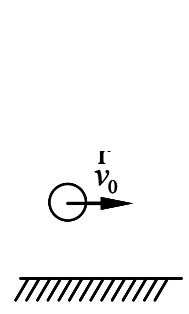

(a)

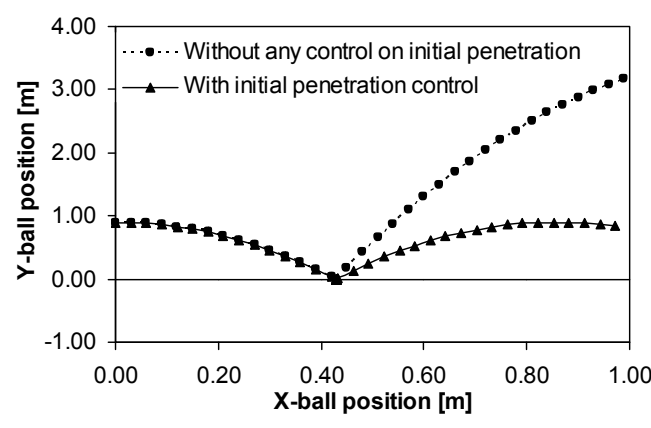

(b)

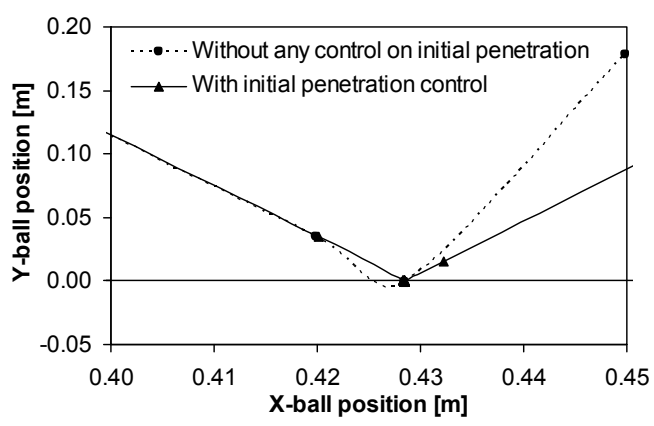

(c)

Figure 1. (a) Initial configuration for a ball falling under the effect of gravity; (b) Trajectory of the falling ball obtained with integration algorithms with and without initial penetration control; (c) Detailed view in the vicinity of contact.

Define by $\delta^{-}$the distance between the two surfaces in the time step $t^{-}$that precedes the time step $t^{+}$, at which contact with a penetration $\delta^{+}$is first detected. In between these time steps, say at $t^{c}$, the penetration $\delta^{c}=0$ exists. Assuming a constant velocity for the multibody system in the vicinity of contact, the time at which contact starts can be calculated by,

$$
t^{c}=t^{-}+\frac{\delta^{-}}{\delta^{+}+\delta^{-}} \Delta t
$$

Consequently, the ideal situation, during the integration of the multibody system of the equations of motion, would be to have a time step in the vicinity of contact of

$$
\Delta t^{\text {ideal }}=t^{c}-t^{-}+\mathcal{E}
$$

where $\varepsilon$ is a very small number to effectively ensure that $\delta_{\max }>\delta^{c} \geq 0$. Several procedures are suggested to ensure that $\delta^{+}<\delta_{\max }$, which can be implemented in any code, depending on the access that exists to its numerical integrator.

Procedure 1: Assume that in the vicinity of contact the motion of the multibody system is such that each body moves approximately with constant velocity. Note that the assumption only needs to be valid within a simple time step. Then the time for contact is calculated by Equation (9) and the ideal time step is calculated using Equation (10). Now, the positions and velocities of the multibody system, at the time of contact, $t^{c}$, are estimated as,

$$
\begin{aligned}
\mathbf{q}^{c} & =\mathbf{q}^{-}+\left(\mathbf{q}^{+}-\mathbf{q}\right) \frac{\Delta t^{\text {ideal }}}{\Delta t} \\
\dot{\mathbf{q}}^{c} & =\dot{\mathbf{q}}^{-}+\left(\dot{\mathbf{q}}^{+}-\dot{\mathbf{q}}\right) \frac{\Delta t^{i d e a l}}{\Delta t}
\end{aligned}
$$

where the superscripts -, + and $c$ mean that the quantity in which they are applied is evaluated at the instant before contact, after contact and at the time of contact, respectively. The integration algorithm is now restarted at time $t^{c}$ with the initial positions and velocities given by Equations (11) and (12). The proposed procedure, being approximate, does present slight violations of the position and velocity constraint equations. Because a constraint stabilization method, or a constraint elimination method, is 
being used it is expected that such violations remain under control. Notice also that when a variable time step integrator is used the restart of the integration process is naturally done with small time steps. Therefore, in the vicinity of contact small time steps are used by the integrator and even if the conditions calculated by Equations (11) and (12) are for an instant just before contact the integration process continues with the guarantee that the initial penetration never exceeds the prescribed threshold.

Procedure 2: The numerical algorithms used for integration of first order differential equations with variable time steps, such as the ones generally used in multibody dynamics [67,68], include an error control that supports the acceptance of rejection of any particular time step. Such decision is based on numerical issues, related to the dynamic response of the system, rather than in any other physical reason. The methodology here proposed ensures that for any contact in a multibody system the time step of the integration is such that any initial penetration is below any prescribed threshold. The numerical error control of the selected integration algorithm is forced to handle the physical criteria to accept/reject time steps in equal terms with the numerical error control that it normally uses. Say that at a given time, during the integration of the multibody system equations of motion, the internal numerical control of the integration algorithm tests a time step $\Delta t_{\text {trial }}$ and decides to accept it. Before it is definitely accepted, the following physical condition must be met by all new contacts detected in the system,

$$
\delta^{(-)}\left(\mathbf{q}, t+\Delta t_{\text {trial }}\right)<\delta_{\max }
$$

where $\delta_{\max }$ is a small positive number that acts as a safeguard against round-off error.

If the condition described by Equation (13) is observed by all new contacts, i.e., contacts that started during the current time step, the integration continues without any further interference. If Equation (13) is not fulfilled, the integration algorithm takes it as an indication to reject the time step and to attempt a smaller time step. Generally such action corresponds to halving the attempted time step, but particular integration error controls may take different actions. When a smaller new time step is attempted the condition defined by Equation (13) is checked again and a decision is made.

Eventually a suitable time step that ensures the fulfillment of Equation (13) for all new contacts is identified. The integrators available in mathematical libraries include features that inform to user if the error control intends to accept or reject a completed time step before doing it. When such features are available the procedure just described is easily implemented without changing anything in the structure or implementation of the integration algorithm. It should be noted that the proposed methodology is used with predictor-corrector algorithms, being the final decision if a particular time step is accepted of rejected is taken after the step is completed. This feature is further discussed in the next section, in which the dynamic analysis of constrained multibody systems including the contact analysis procedure proposed here is presented. 


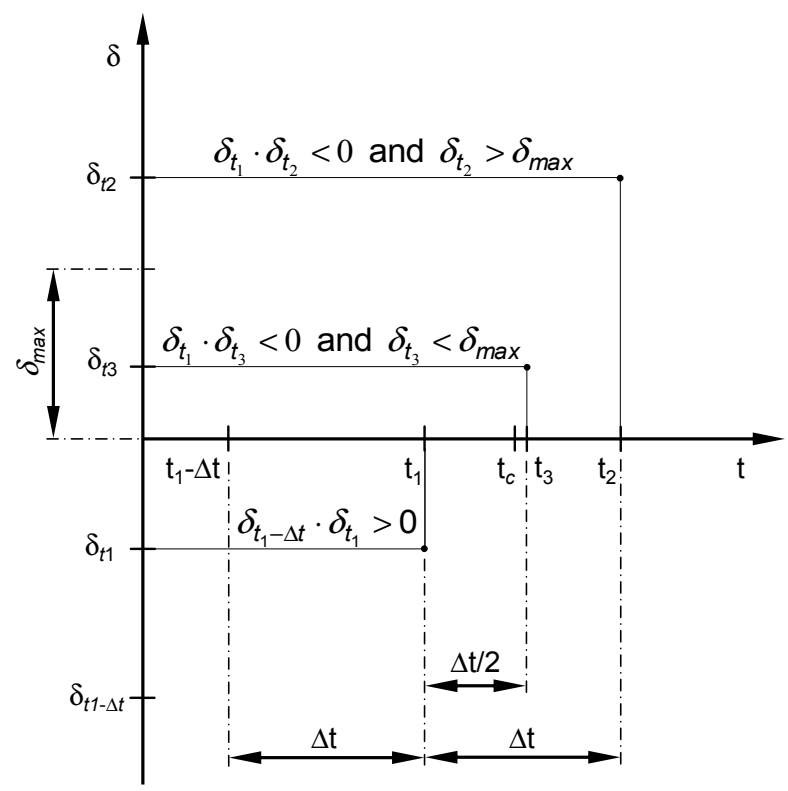

Figure 2. Computational scheme to determine the exact instant of contact.

In short, if Equation (13) is satisfied, then the current time is taken as the impact time. If Equation (13) is not satisfied it is because the current time step is far past the impact time. In this case, the integration algorithm receives the information that the time step has to be rejected so that a new time step is attempted according to the strategy used by the internal control of the integrator. This procedure is repeated until both Equations (8) and (13) are verified. This computational strategy to determine the instant of contact is schematically illustrated in Figure 2, where $t_{c}$ denotes the exact contact time and $t_{1}, t_{2}$ and $t_{3}$ represent three different "current" times. It should be highlighted that with this methodology, due to the possibility that the user imposes penetration tolerances that are too small, the step size can reach smaller values than those required to keep the integration tolerance error under control and the numerical system can become unstable. As a safeguard, when the step size tries to go below a given limit, due to the penetration tolerance imposed, it is forced to remain at the minimum value of $10^{-7} \mathrm{~s}$.

\section{Equations of Motion for Constrained Multibody Systems}

Using Cartesian coordinates, the equations of motion for planar multibody mechanical systems are written as a coupled set of differential and algebraic equations expressed as [65],

$$
\left[\begin{array}{cc}
\mathbf{M} & \boldsymbol{\Phi}_{\mathbf{q}}^{T} \\
\boldsymbol{\Phi}_{\mathbf{q}} & \mathbf{0}
\end{array}\right]\left\{\begin{array}{c}
\ddot{\mathbf{q}} \\
\boldsymbol{\lambda}
\end{array}\right\}=\left\{\begin{array}{l}
\mathbf{g} \\
\boldsymbol{\gamma}
\end{array}\right\}
$$

where $\mathbf{M}$ is the system mass matrix, $\boldsymbol{\Phi}_{\mathbf{q}}$ is the Jacobian matrix of the constraint equations, $\ddot{\mathbf{q}}$ is the vector that contains the system accelerations, $\lambda$ is the vector that contains unknown Lagrange multipliers associated with kinematic constraints, $\mathbf{g}$ is the generalized force vector and $\boldsymbol{\gamma}$ is the right 
hand side of the acceleration equations, which contains the terms that are exclusively function of velocity, position and time. This system of equations is solved for $\ddot{\mathbf{q}}$ and $\boldsymbol{\lambda}$. Then, in each integration time step, the accelerations vector, $\ddot{\mathbf{q}}$, together with velocities vector, $\dot{\mathbf{q}}$, are integrated in order to obtain the system velocities and positions for the next time step. This procedure is repeated until the final of the analysis time is reached. Note that the equations of motion may exhibit a stiff behavior, and therefore, integration algorithms such as the one proposed by Gear [68] are preferred for the numerical solution of the problem. The Baumgarte stabilization method [66], or any other [67], may be used to ensure the stabilization or the elimination of the constraint violation associated with the kinematic constraints of the ideal joints.

In what follows, main numerical aspects related to the standard integration of the equations of motion of a constrained multibody system are reviewed with the purpose of better understanding how to include the methodology that controls the identification of the impact time. The standard integration of the equations of motion converts the $n c$ second order differential equations of motion into $2 n c$ first order differential equations. Then, a numerical scheme is employed to solve the initial value problem $[68,69]$. The commonly used numerical integration algorithms are useful in solving first-order differential equations that take the form [69],

$$
\dot{\mathbf{y}}=f(\mathbf{y}, t)
$$

Thus, if there are $n c$ second order differential equations, they are converted to $2 n c$ first order equations by defining the $\mathbf{y}$ and $\dot{\mathbf{y}}$ vectors, which contains, respectively, the system positions and velocities and the system velocities and accelerations as

$$
\mathbf{y}=\left\{\begin{array}{c}
\mathbf{q} \\
\dot{\mathbf{q}}
\end{array}\right\} \quad \text { and } \quad \dot{\mathbf{y}}=\left\{\begin{array}{c}
\dot{\mathbf{q}} \\
\ddot{\mathbf{q}}
\end{array}\right\}
$$

The numerical integration at instant of time $t$ that leads to the calculation of the state variables at $t+\Delta t$ is described as

$$
\dot{\mathbf{y}}(\mathrm{t}) \stackrel{\text { Integration }}{\longrightarrow} \mathbf{y}(\mathrm{t}+\Delta \mathrm{t})
$$

which means that velocities and accelerations at instant $t$ are integrated to yield positions and velocities at next time step, $t=t+\Delta t$. Figure 3 presents a flowchart of computational strategy for dynamic analysis of constrained multibody systems including contact analysis procedure presented in the previous section, which can be summarized by the following steps:

i) Start at instant of time $t^{0}$ with given initial conditions for positions $\mathbf{q}^{0}$ and velocities $\dot{\mathbf{q}}^{0}$.

ii) Assemble the global mass matrix $\mathbf{M}$, evaluate the Jacobian matrix $\boldsymbol{\Phi}_{\mathbf{q}}$, construct the constraint equations $\boldsymbol{\Phi}$, determine the right hand side of the accelerations $\gamma$, and calculate the force vector $\mathbf{g}$. 
iii) Solve the linear set of the equations of motion (14) for a constrained mechanical system in order to obtain the accelerations $\ddot{\mathbf{q}}$ at time $t$ and the Lagrange multipliers $\lambda$.

iv) Assemble the vector $\dot{\mathbf{y}}_{t}$ containing the generalized velocities $\dot{\mathbf{q}}$ and accelerations $\ddot{\mathbf{q}}$ for instant of time $t$.

v) Integrate numerically the $\underset{t}{\&}$ and $\underset{t}{\boldsymbol{q}}$ for time step $t+\Delta t$ and obtain the new positions $\mathbf{q}_{t+\Delta t}$ and velocities $\&_{t+\Delta t}$. After the time step is completed, i.e., after passing the time step acceptance criteria defined by the internal error control of the integration algorithm, an enquire is issued to accept or not the integration time step. The suggested positions and velocities associated to the completed time-step are available for the confirmation if any new contact is started and if the associated penetration depth is acceptable.

vi) Check for contact between contacting surfaces and: (a) if no new contact is detected, accept the time step and proceed to step viii) of this procedure; (b) if there is at least one new contact and all penetration depths of the contacts that started during the current time step are below the penetration tolerance, accept the time step proceed to step viii) of this procedure; (c) if there is at least one new contact, started during the current time step, for which the initial penetration exceeds the penetration tolerance proceed.

vii) If the current time step is larger than the minimum time step threshold, say $10^{-7} \mathrm{~s}$, inform the integration algorithm that the time step must be rejected and return to step v) of this procedure. If the time step being used is smaller or equal to the minimum time step threshold just warn the user that the initial penetration may be excessive and proceed.

viii) Update the time variable and go to step ii) if the current time of analysis is smaller than the intended time of simulation. Otherwise, terminate the analysis.

The procedure just described it is easy to implement in numerical integrators for which the source code is available, such as the DE/STEP integrator by Shampine and Gordon [69]. If the source code of the integrator is not available the proposed procedure can only be used if the integrator informs the user if it intends to accept or reject a particular time step and allows the user to override the decision of its error control. The integrator DIVPAG included in the IMSL Library [70], in which the AdamsMoulton and the Gear's BDF methods are implemented, includes the enquire features that allow for the decision to accept or reject the time step to be overridden. Other integration algorithms included in numerical methods libraries or in development environments, such as Matlab [71], must be checked for the availability of the features that allow using the proposed methodology or for the existence of alternative characteristics that may serve the same purpose. 


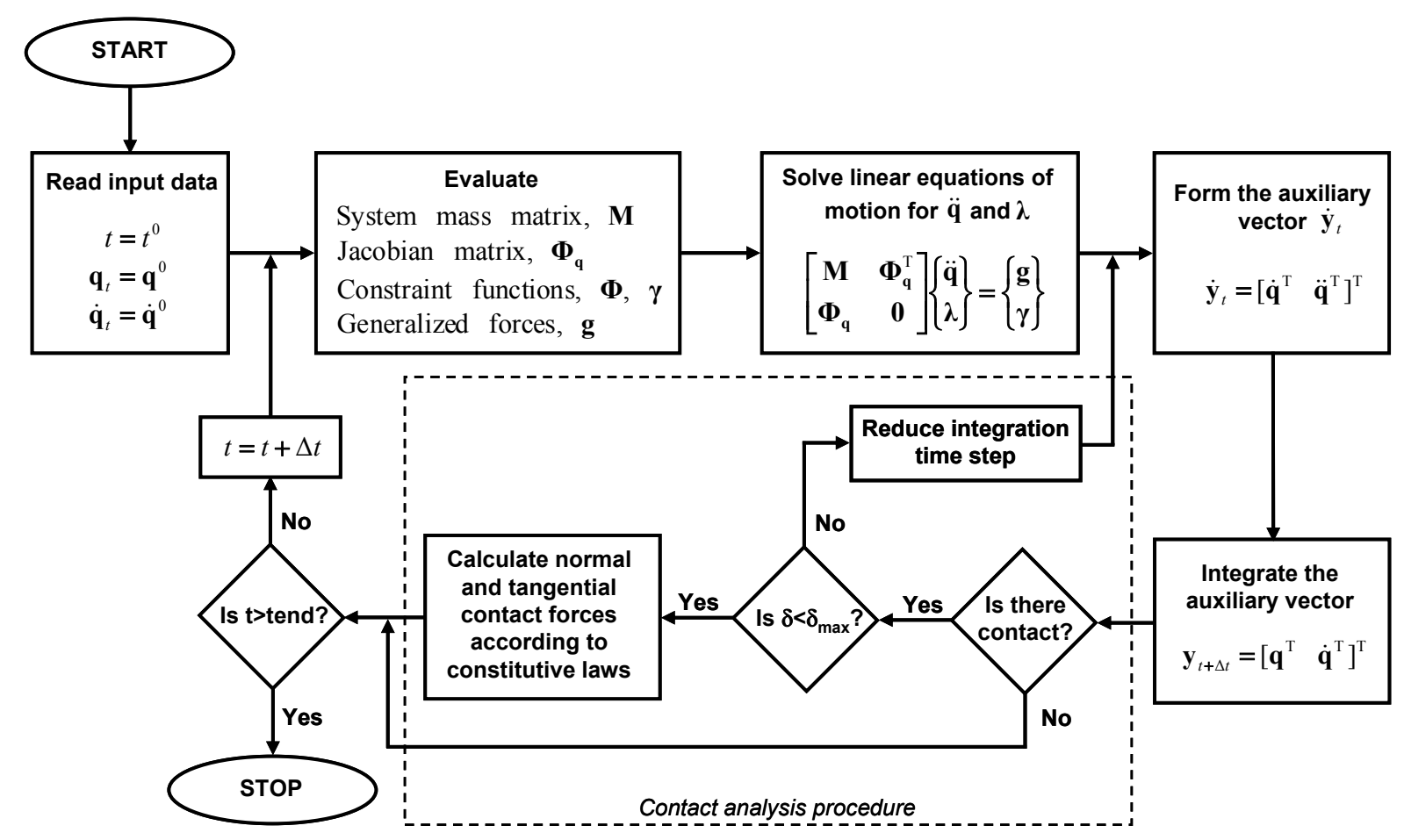

Figure 3. Flowchart of computational procedure for dynamic analysis of constrained multibody systems including contact analysis procedure.

\section{Demonstrative Applications to a Simple Pendulum and a Quick-Return Mechanism}

\subsection{Simple Pendulum}

A sphere with a radius of $0.05 \mathrm{~m}$ is attached to the extremity of the arm forming the pendulum shown in Figure 4. The pendulum arm is released from the initial configuration under the gravity action only, which is taken in the negative $Y$ direction, and hits the rigid wall. The contact forces between the sphere and wall are evaluated by using Equation (6) with fully elastic restitution coefficient, a generalized stiffness parameter $K$ of $3.39 \times 10^{10} \mathrm{~N} / \mathrm{m}^{1.5}$ and an exponent $n$ of 1.5 .

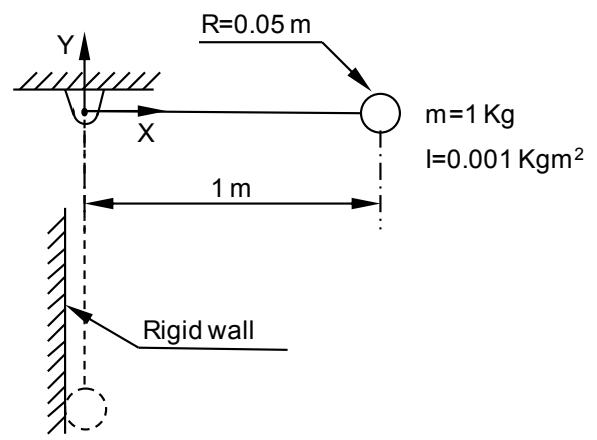

Figure 4. Simple pendulum colliding against a rigid wall.

The kinematics of the sphere is described by the graphs shown in Figure 5. Since the contact is fully elastic, when the initial control penetration is active, the sphere rebounds to its initial position after it collides with the wall. Conversely, when there is no initial penetration control during the numerical 
integration the sphere rebounds to a higher height than its initial configuration. This physically inconsistent behavior is justified by an increase of energy during the contact associated to the high value of the initial contact penetration, which, in turn, leads to a high restitution force.

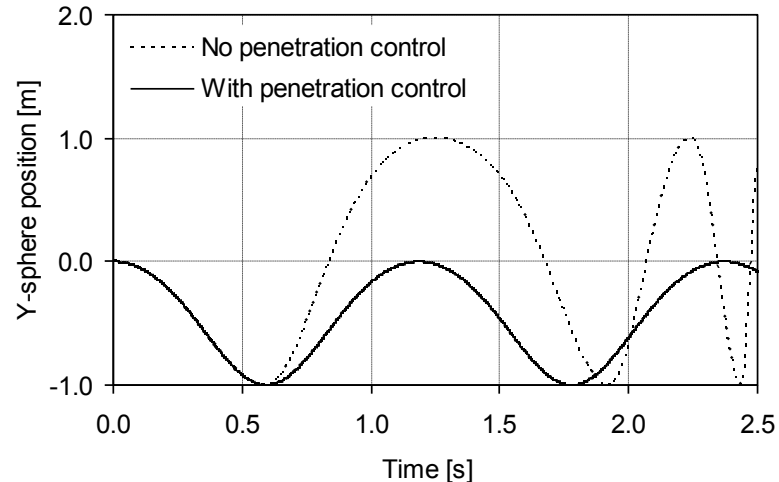

(a)

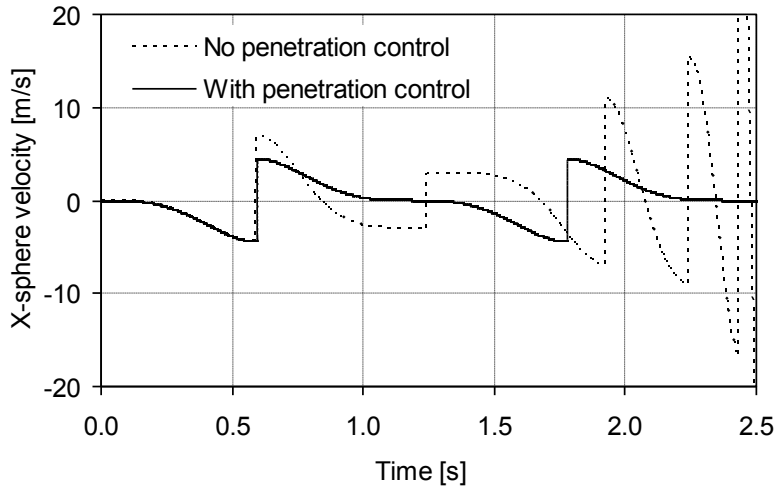

(b)

Figure 5. Kinematics of the sphere: (a) position in $Y$; (b) Velocity in $X$.

With the intent to better understand what happens during penetration with and without the time step control, the variation of the time step is plotted in Figure 6. Together with the evolution of the penetration, Figure 6 clearly shows that a new time step starts at the precise instant of the start of contact. Notice the decrease of time step just before the start of contact and its increase up to its original value after the contact is over, which in the present case is $10^{-4} \mathrm{~s}$. With this approach it is possible to use larger time steps when there is no contact, being the time step reduced based on physical criterion related to the initial penetration tolerance, only if contact is detected.

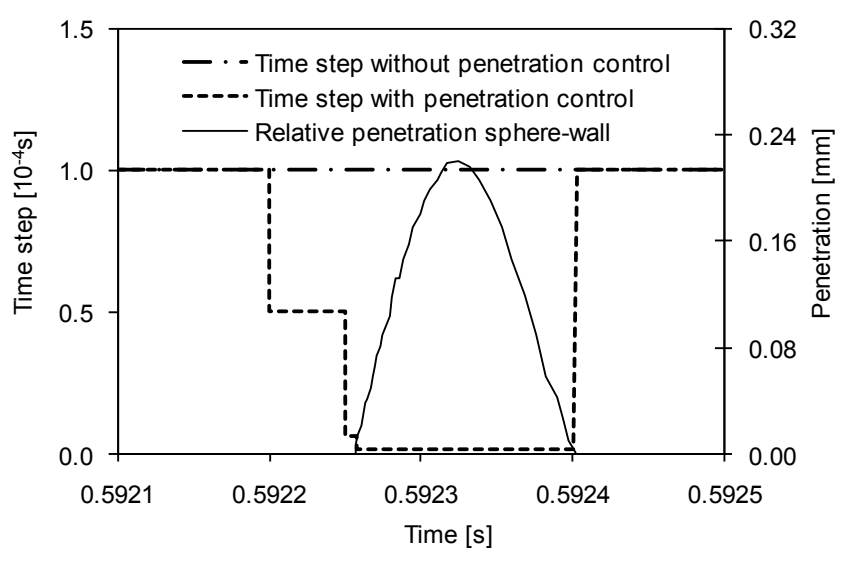

Figure 6. Time step adjustment with the control on the initial penetration.

The pendulum is also simulated using a commercial program for general mechanical systems. Simulations are performed with time steps of $0.0200,0.0175$ and $0.0150 \mathrm{~s}$, referred by SIM-I, SIM-II and SIM-III respectively, being the sequences of time frames for the three scenarios shown in Figure 7. Simulations are showing dramatically different results or become even unstable depending on the time step. 


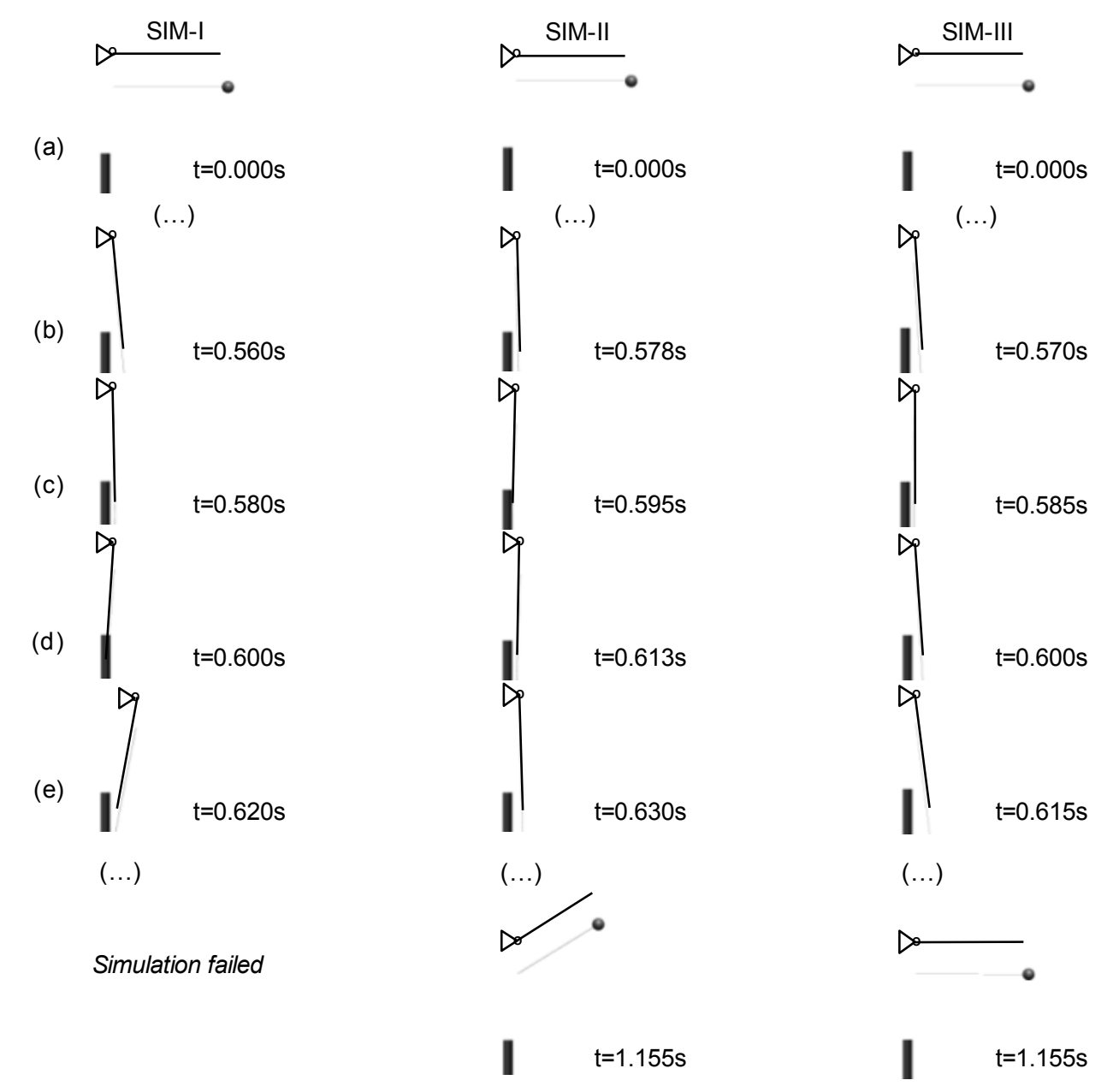

Figure 7. Animation sequence for three simulations obtained using a commercial program.

\subsection{Quick Return Mechanism}

The application of the proposed methodology to a more complex multibody system is represented by the simulation of a quick-return mechanism. This mechanism is made of six rigid bodies, one ideal revolute joint between the ground and crank, two perfect translational joints and four revolute clearance joints, as illustrated in Figure 8. Due to the existence of the four revolute clearance joints, the system has a total of nine degrees of freedom. The acceleration due to gravity is taken as acting in the negative $Y$ direction and the system is defined as moving the $X Y$ plane. The set of data adopted for the model is listed in Table I.

Table I. Geometric and inertia data of the quick-return mechanism.

\begin{tabular}{cccc}
\hline Body Nr. & Length $[\mathrm{mm}]$ & Mass $[\mathrm{Kg}]$ & Moment of inertia $\left[\mathrm{kgm}^{2}\right]$ \\
\hline 2 & 100 & 0.20 & 0.010 \\
3 & 500 & 1.00 & 0.100 \\
4 & - & 0.50 & 0.025 \\
5 & 120 & 0.24 & 0.012 \\
6 & - & 0.50 & 0.025 \\
\hline
\end{tabular}

The quick-return mechanism, which produces a slow cutting stroke of a tool and a rapid return stroke, is driven by a rotational motor attached to the crank rotating with a constant angular velocity of 
$3 \mathrm{rad} / \mathrm{s}$. The remaining initial conditions, necessary to start the dynamic analysis, are obtained from the kinematic simulation of the quick-return mechanism in which all the joints are modeled as ideal joints. The parameters used for the dynamic simulation are listed in Table II. A radial clearance of $0.5 \mathrm{~mm}$ is used in all clearance joints.

Table II. Parameters used in the dynamic simulation for the quick-return mechanism.

\begin{tabular}{llll}
\hline Bearing radius & $10.0 \mathrm{~mm}$ & Young's modulus & $207 \mathrm{GPa}$ \\
Journal radius & $9.5 \mathrm{~mm}$ & Poisson's ratio & 0.3 \\
Restitution coefficient & 0.9 & Integration step & $0.0001 \mathrm{~s}$ \\
\hline
\end{tabular}

Each clearance joint is modeled as two colliding bodies, being the journal freely to move inside the bearing boundaries. The occurrence of contact within the clearance joints is determined by evaluating the relative penetration at any time during the numerical solution of the system dynamics as,

$$
\delta_{i}=e_{i}-c_{r i} \quad(i=1, \ldots, 4)
$$

where $e_{i}$ is the relative eccentricity between the journal and bearing centers and $c_{r i}$ is the radial clearance. The computational modeling of the contact between journal and bearing, within the clearance revolute joints, uses continuous contact force model with the energy dissipation in form of hysteresis damping, given by Equation (6). For the interested reader the complete formulations on the revolute clearance joints, in the context of multibody systems, are presented in the work by Flores et al. [5].

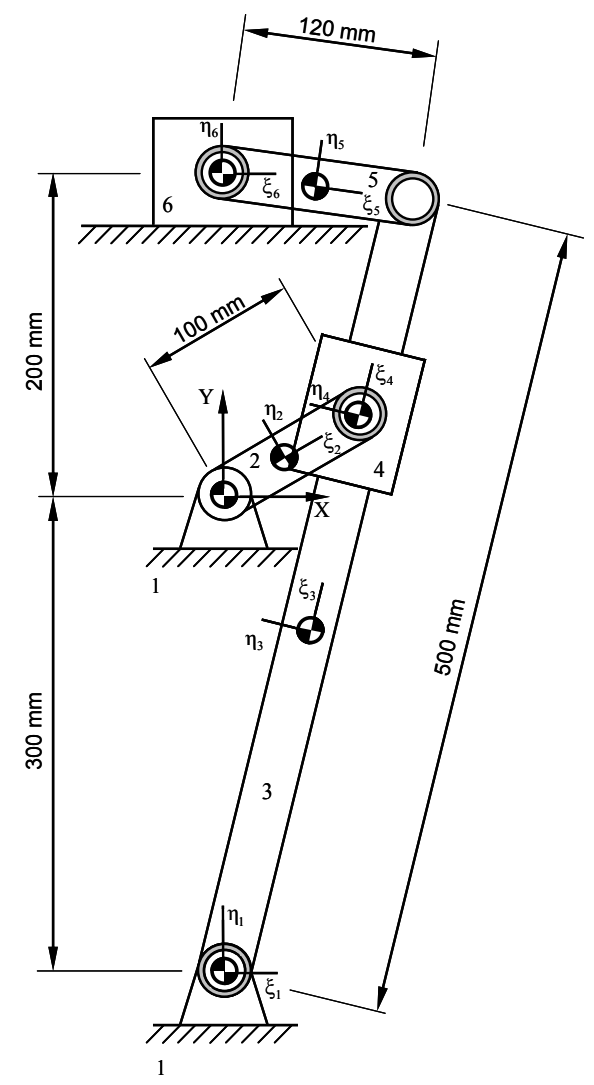

Figure 8. Quick-return mechanism with four revolute clearance joints. 
The performance of the quick-return mechanism is quantified by plotting the $X$ position and velocity components of the slider, represented by body 6 . This allows comparing the results obtained with the current model to the dynamic behavior of the mechanism with ideal joints simulated with the same conditions. Additionally, the relative penetration and contact force produced during one arbitrary contact, between journal and bearing in the revolute clearance joint that connects the crank and slider represented by body 4 , is also plotted.

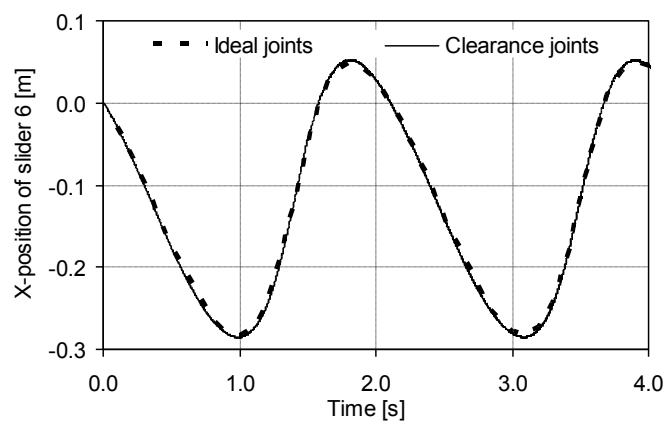

(a)

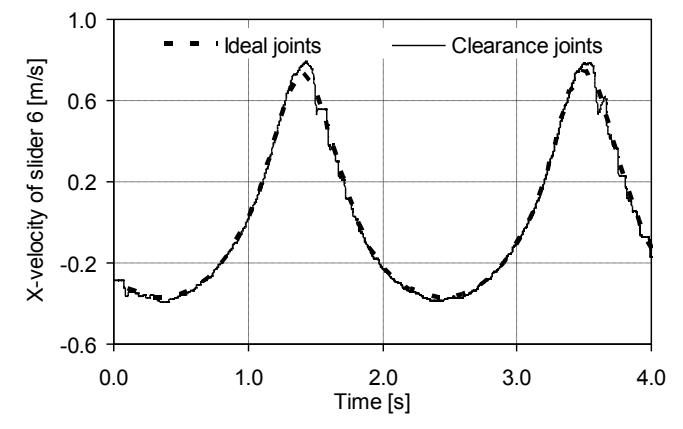

(b)

Figure 9. (a) $X$-position of the slider, represented by body 6; (b) $X$-velocity of the slider.

Figure 9 shows how the clearance joints influence the position and velocity of the slider. The horizontal line segments in the velocity-time curve correspond to constant slider velocity, which means that there is no contact between the journal and bearing surfaces while such velocity is constant. Sudden changes in the velocity are clearly associated with the impacts within the clearance joints, which are quite visible by the step shaped curve of the velocity diagram. Smooth changes in the velocity can also be observed indicating that the journal and bearing surfaces are in permanent or continuous contact, that is, the journal follows the bearing wall.

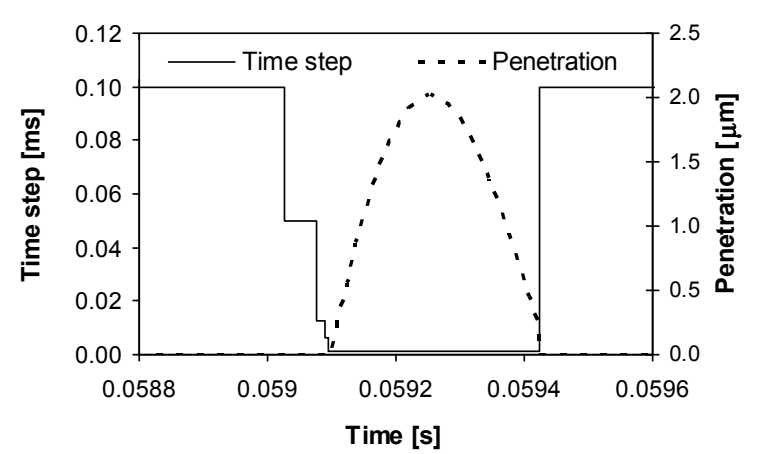

(a)

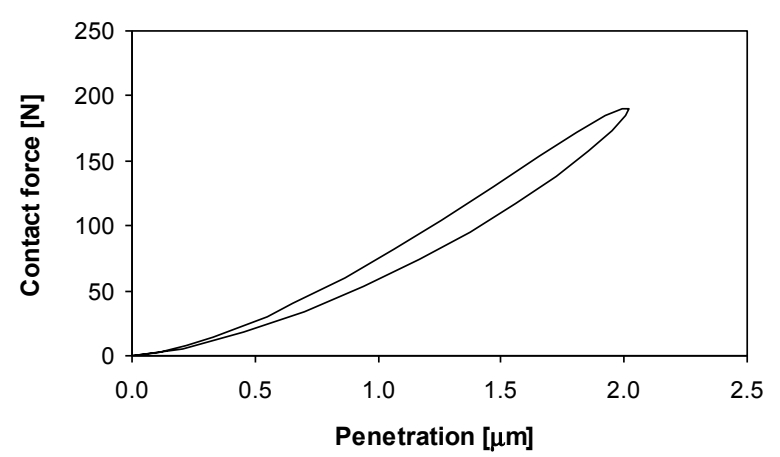

(b)

Figure 10. (a) Relative penetration; (b) Contact force.

Figure 10 illustrates the penetration and contact force produced during one arbitrary impact between the journal and bearing of the revolute clearance joint that connects the crank and slider, represented by body 4 . The variation of the time step is also shown in Figure 10(a), where it is clear that it is adjusted, in the vicinity of contact such a way that the start of contact coincides with the start 
of a new time step. It must be referred that when the quick-return mechanism is simulated without any control on the initial contact penetration, the first impact takes place with a relative penetration of $0.0012 \mathrm{~m}$, which corresponds to a contact force equal to $3000000 \mathrm{~N}$ being impossible to proceed with the numerical simulation of the mechanism, i.e., the integration algorithm simply fails and the simulation aborts. Such initial penetrations and their corresponding contact forces are physically impossible and, consequently, the quick-return mechanism experiences such high variations of the dynamic forces that the integration algorithm perceives them as high frequency response contents and tries to adjust the time step accordingly, ultimately stalling the whole process.

In order to better understand what occurs during the dynamic simulation of the quick-return mechanism with control on initial penetration, consider Figure 11, in which the journal center orbit inside the bearing boundaries is illustrated. The different types of relative motion between the journal and bearing are observed, namely, the free flight motion, the impact followed by rebound and the permanent or continuous contact motion. The relative penetration is visible by the points plotted outside the clearance circle. A point is plotted for each integration time step. The point density in Figure 11 is very high when the journal contacts the bearing, which means that the step size is small. When the journal is in free flight motion the time step is increased and, consequently, the dots plotted in the Figure 11 are further apart.

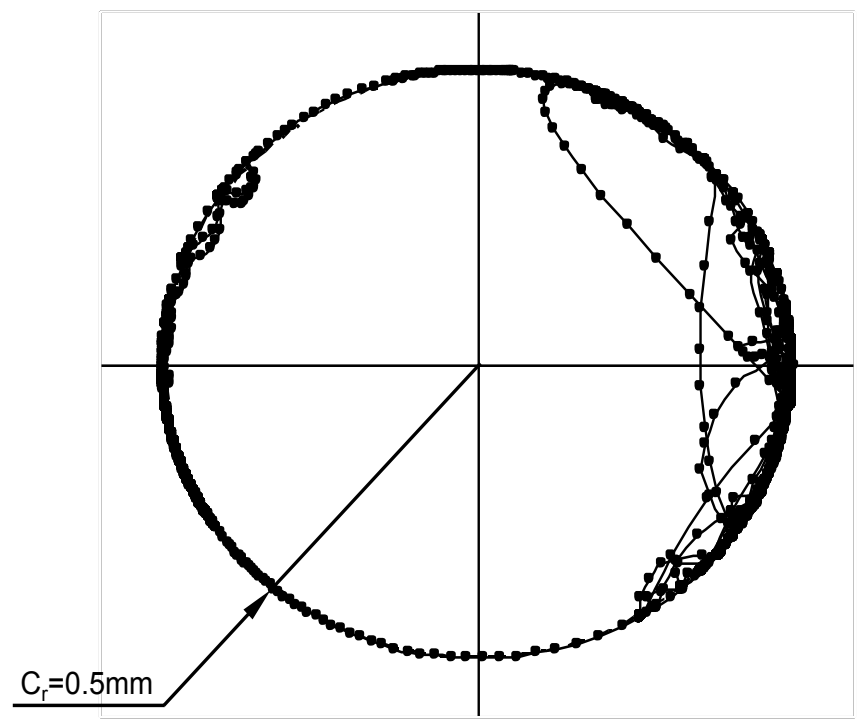

Figure 11. Journal center orbit inside the bearing boundaries.

\section{Conclusions}

A general methodology for the automatic detection of precise instant of contact in contactimpact analysis in multibody dynamics, and for adjusting the integration time step accordingly, has 
been presented in this work. Special emphasis was put on the use of a continuous contact force model, which explicitly accounts for the deformation of the bodies during the impact process.

The fundamental idea behind the methodology proposed is to ensure that the start of impact between any pair of contacting surfaces in a multibody system does not occur with a large initial penetration and, in the process, to avoid the artificially large contact forces that develop. The detection of the precise instant of contact takes place when the distance between two bodies changes sign between two discrete moments in time, i.e., two successive time steps. Thus, if during the numerical integration of the system equations of motion any penetration that is detected to start with a depth below a previously specified tolerance, the current time is taken as the impact time. However, if the initial penetration is larger than the specified tolerance it means that the current time step is beyond the impact time. In this case, integration algorithm is forced to go back and take a smaller time step. The process progresses only if a time step leading to an initial penetration within the acceptable tolerance can be taken.

The methodology proposed has been implemented computationally and applied to the simulation of the multibody systems of a simple pendulum and a quick-return mechanism, which includes four revolute joints with clearances. The demonstration cases show that the lack of control for the depth of the initial penetration of the contact leads either to unfeasible results of the dynamic simulation, characterized by gains of energy in the system, or to a stall and eventual failure of the integration process. Even when correct results are obtained when no penetration control is used in simple problems such is due to chance rather than by any mechanically based decision. It has also been shown that the decision on the size of the reduction of the time step, taken by the integration algorithm, is solely done by the numerical control originally implemented and not by the user. In this form the accuracy of the numerical integration methodology is not affected because no changes on the integration algorithm are required.

\section{Acknowledgements}

This work is supported by the Portuguese Foundation for the Science and Technology under the research project BIOJOINTS (PTDC/EME-PME/099764/2008). The authors would also like to acknowledge the anonymous reviewers for their insightful comments and suggestions on an earlier draft of this paper.

\section{References}

1. Moreau, J.J., 'Numerical aspects of the sweeping process', Computers Methods in Applied Mechanics and Engineering, 177, 1999, 329-349.

2. Gonthier, Y., McPhee, J., Lange, C., Piedboeuf, J-C., 'A regularized contact model with asymmetric damping and dwell-time dependent friction', Multibody System Dynamics, 11, 2004, 209-233.

3. Glocker, C., Studer, C., 'Formulation and preparation for numerical evaluation of linear complementary systems in dynamics', Multibody System Dynamics, 13, 2005, 447-463.

4. Sharf, I., Zhang, Y., 'A contact force solution for non-colliding contact dynamics simulation', Multibody System Dynamics, 16, 2006, 263-290. 
5. Flores, P., Ambrósio, J., Claro, J.P., 'Dynamic Analysis for Planar Multibody Mechanical Systems with Lubricated Joints', Multibody System Dynamics, 12, 2004, 47-74.

6. Najafabadi, S.A.M., Kövecses, J., Angeles, J., 'Impacts in multibody systems: modeling and experiments', Multibody System Dynamics, 20, 2008, 163-176.

7. $\quad$ Pfeifer. F., Glocker C., Multibody dynamics with unilateral constraints, John Wiley and Sons, New York, 1996.

8. Wriggers, P., Computational Contact Mechanics, Second Edition. Springer-Verlag Berlin, 2006.

9. Qiang, T., Zhang, Y., Chen, L., Flores, P., 'Dynamics of spatial flexible multibody systems with clearance and lubricated spherical joints', Computers and Structures, 87(13-14), 2009, 913-929.

10. Förg, M., Pfeiffer, F., Ulbrich, H., Simulation of Unilateral Constrained Systems with Many Bodies, Multibody System Dynamics, 14(2), 2005, 137-154.

11. Ambrósio, J., Verissimo, P., Improved bushing models for general multibody systems and vehicle dynamics, Multibody System Dynamics, 22, 2009, 341-365.

12. Machado, M., Flores, F., Claro, J.C.P., Ambrósio, J., Silva, M., Completo, A., Lankarani, H.M., Development of a planar multibody model of the human knee joint, Nonlinear Dynamics, 2010, DOI 10.1007/s11071-009-9608-7.

13. Choi, J., Ryu, H.S., Kim, C.W., Choi, J.H., An efficient and robust contact algorithm for a compliant contact force model between bodies of complex geometry, Multibody System Dynamics, 23, 2010, 99-120.

14. Flores, P., Lankarani, H.M., Spatial rigid-multibody systems with lubricated spherical clearance joints: modeling and simulation, Nonlinear Dynamics, 2010, DOI 10.1007/s11071-009-9583-z.

15. Erkaya, S., Uzmay, I., Experimental investigation of joint clearance effects on the dynamics of a slider-crank mechanism, Multibody System Dynamics, 2010, DOI 10.1007/s11044-010-9192-0.

16. Gilardi, G., Sharf, I., 'Literature survey of contact dynamics modelling', Mechanism and Machine Theory, 37, 2002, 1213-1239.

17. Flores, P., Ambrósio, J., Claro, J.C.P., Lankarani, H.M., 'Influence of the contact-impact force model on the dynamic response of multibody systems', Proceedings of the Institution of Mechanical Engineers, Part-K Journal of Multi-body Dynamics, 220(1), 2006, 21-34.

18. Han, I., Gilmore, B.J., 'Multi body impact motion with friction analysis, simulation, and validation', Journal of Mechanical Design, 115, 1993, 412-422.

19. Haug, E.J., Wu, S.C., Yang, S.M., 'Dynamics of mechanical systems with coulomb friction, stiction, impact and constraint addition deletion - I Theory', Mechanism and Machine Theory, 21, 1986, 401-406.

20. Djerassi, S., 'Collision with friction; Part A: Newton's hypothesis', Multibody System Dynamics, 21, $2009,37-54$.

21. Bowling, A., Flickinger, D.M., Harmeyer, S., Energetically consistent simulation of simultaneous impacts and contacts in multibody systems with friction, Multibody System Dynamics, 22, 2009, 27-45.

22. Flickinger, D.M., Bowling, A., Simultaneous oblique impacts and contacts in multibody systems with friction, Multibody System Dynamics, 23, 2010, 249-261.

23. Flores, P., Leine, R., Glocker, C., Modeling and analysis of planar rigid multibody systems with translational clearance joints based on the non-smooth dynamics approach, Multibody System Dynamics, 23, 2010, 165-190.

24. Dimitrakopoulos, E.G., Analysis of a frictional oblique impact observed in skew bridges, Nonlinear Dynamics, 2010, DOI 10.1007/s11071-009-9616-7.

25. Bhalerao, K.D., Anderson, K.S., Modeling intermittent contact for flexible multibody systems, Nonlinear Dynamics, 2010, DOI 10.1007/s11071-009-9580-2.

26. Djerassi, A., Collision with friction; Part B: Poisson's and Stornge's hypotheses, Multibody System Dynamics, 21, 2009, 55-70.

27. Lee, T.W., Wang, A.C., 'On the dynamics of intermittent-motion mechanisms. Part 1 - Dynamic model and response', Journal of Mechanisms, Transmissions, and Automation in Design, 105, 1983, 534-540.

28. Khulief, Y.A., Shabana, A.A., 'Dynamic analysis of constrained system of rigid and flexible bodies with intermittent motion', Journal of Mechanisms, Transmissions, and Automation in Design, 108, 1986, 38-45.

29. Khulief, Y.A., Shabana, A.A., 'A continuous force model for the impact analysis of flexible multibody systems', Mechanism and Machine Theory, 22, 1987, 213-224.

30. Flores, P., Ambrósio, J., 'Revolute joints with clearance in multibody systems', Computers and Structures, 82, 2004, 1359-1369.

31. Flores, P., 'Modeling and simulation of wear in revolute clearance joints in multibody systems', Mechanism and Machine Theory, 44(6), 2009, 1211-1222.

32. Acary, V., Brogliato, B., Numerical Methods for Nonsmooth Dynamical Systems: Applications in Mechanics and Electronics. Lecture Notes in Applied and Computational Mechanics Vol. 35, Berlin Heidelberg New-York, Springer-Verlag, 2008. 
33. Erickson, D., Weber, M., Sharf, I., Contact Stiffness and Damping Estimation for Robotic Systems, The International Journal of Robotics Research, 22(1), 2003, 41-57.

34. Sousa, L., Veríssimo, P., Ambrósio, J., Development of generic multibody road vehicle models for crashworthiness, Multibody System Dynamics, 19, 2008, 133-158.

35. Carsten, H., Wriggers, P., An Explicit Multi-Body Contact Algorithm, Proc. Appl. Math. Mech., 3, $2003,280-281$.

36. Hippmann, G., An Algorithm for Compliant Contact Between Complexly Shaped Bodies, Multibody System Dynamics, 12, 2004, 345-362.

37. Ebrahimi, S., Hippmann, G., Eberhard, P., Extension of polygonal contact model for flexible multibody systems, Int. J. of Appl. Math. and Mech., 1, 2005, 33-50.

38. Ebrahimi, S., Eberhard, P., A linear complementarity formulation on position level for frictionless impact of planar deformable bodies, ZAMM Z. Angew. Math. Mech., 86(10), 2006, 807-817.

39. He, K., Dong, S., Zhou, Z., Multigrid contact detection method, Physical Review, 75(3), $2007,036710$.

40. Wellmann, C., Lillie, C., Wriggers, P., A contact detection algorithm for superellipsoids based on the commonnormal concept, Engineering Computations: International Journal for Computer-Aided Engineering and Software, 25(5), 2008, 432-442.

41. Studer, C., Leine, R.I., Glocker, C., (2008) Step size adjustment and extrapolation for time-stepping schemes in nonsmooth dynamics. International Journal for Numerical Methods in Engineering 76(11) 1747-1781

42. Portal, R.J.F., Dias, J.M.P., Sousa, L.A.G., Contact detection between convex superquadric surfaces on multibody dynamics, Proceedings of the Multibody Dynamics 2009, ECCOMAS Thematic Conference, K. Arczewski, J. Frączek, M. Wojtyra (eds.), Warsaw, Poland, 29 June - 2 July 2009, 14p.

43. Lankarani, H.M., Nikravesh, P.E., 'A contact force model with hysteresis damping for impact analysis of multibody systems', Journal of Mechanical Design, 112, 1990, 369-376.

44. Glocker, C., Set-Valued Force Laws: Dynamics of Non-Smooth Systems. Lecture Notes in Applied Mechanics 1, Springer-Verlag, Berlin, 2001.

45. Flores, P., Ambrósio, J., Claro, J.C.P., Lankarani, H.M., 'Translational Joints With Clearance in Rigid Multibody Systems', Journal of Computational and Nonlinear Dynamics, 3(1), 2008, 0110071-10

46. Lankarani, H.M., Nikravesh, P.E., 'Continuous Contact Force Models for Impact Analysis in Multibody Systems', Nonlinear Dynamics, 5, 1994, 193-207.

47. Dias, J.M.P., Pereira, M.S., 'Dynamics of Flexible Mechanical Systems with Contact-Impact and Plastic Deformations', Nonlinear Dynamics, 8, 1995, 491-512.

48. Hunt, K.H., Crossley, F.R., 'Coefficient of restitution interpreted as damping in vibroimpact', Journal of Applied Mechanics, 7, 1975, 440-445.

49. Oden, J.T., Martins, J.A.C., 'Models and Computational Methods for Dynamic Friction Phenomena', Computer Methods in Applied Mechanics and Engineering, 52, 1985, 527-634.

50. Feeny, B., Guran, A., Hinrichs, N., Popp, K., 'A Historical Review on Dry Friction and Stick-Slip Phenomena', Applied Mechanics Review, 51, 1998, 321-341.

51. Brogliato, B., Ten Dam, A.A., Paoli, L., Genot, F., Abadie, M., 'Numerical simulations of finite dimensional multibody nonsmooth mechanical systems', Applied Mechanics, 55, 2002, 107-150.

52. Glocker, C., Pfeiffer, F., 'Complementarity Problems in Multibody Systems with Planar Friction', Archive of Applied Mechanics, 63(7), 1993, 452-463.

53. Pang, J., Trinkle, J.C., 'Complementarity formulations and existence of solutions of dynamic multi-rigid-body contact problems with Coulomb friction', Mathematical Programming, 73(2), 1996, 199-226.

54. Trinkle, J.C,, Tzitzouris, J.A., Pang, J.S., 'Dynamic multi-rigid-body systems with concurrent distributed contacts', Philosophical Transactions: Mathematical, Physical and Engineering Sciences, 359(1789), 2001,2575-2593

55. Pfeiffer, F., 'The idea of complementarity in multibody dynamics', Archive of Applied Mechanics, 72(11-12), 2003, 807-816.

56. Signorini A, Sopra alcune questioni di elastostatica, Atti della Societa Italian per il Progresso della Scienza, 1933.

57. Moreau, J.J., 'Application of convex analysis to some problems of dry friction', Trends in Applications of Pure Mathematics to Mechanics, (H. Zorski, Ed.) London, U.K.: Pitman, 2, 1979, 263-280.

58. Panagiotopoulos, P.D., Inequality Problems in Mechanics and Applications. Convex and Nonconvex Energy Functionals. Birkhäuser-Verlag., Basel, Boston, Stuttgart, 1985.

59. Kwak, B.M., 'Complementarity Problem Formulation of Three-Dimensional Frictional Contact', Journal of Applied Mechanics, 58, 1991, 134-140.

60. Anitescu, M., Potra, F.A., 'Formulating Dynamic Multi-Rigid-Body Contact Problems with Friction as Solvable Linear Complementarity Problems', Nonlinear Dynamics, 14(3), 1997, 231-247. 
61. Brogliato, B., 'Some perspectives on the analysis and control of complementarity systems', IEEE Transactions on Automatic Control, 48(6), 2003, 918-935.

62. Leine, R.I., Nijmeijer, H., Dynamics and Bifurcations of Non-Smooth Mechanical Systems, Lecture Notes in Applied and Computational Mechanics Vol. 18, Berlin Heidelberg New-York, Springer-Verlag, 2004.

63. Hertz, H., On the contact of solids - On the contact of rigid elastic solids and on hardness, (Translated by D. E. Jones and G. A. Schott), Miscellaneous Papers, Macmillan and Co. Ltd., London, England, p. 146-183, 1896.

64. Goldsmith, W., Impact - The theory and physical behaviour of colliding solids, Edward Arnold Ltd, London, England, 1960.

65. Nikravesh, P., Computer-aided analysis of mechanical systems, Prentice Hall, Englewood Cliffs, New Jersey, 1988.

66. Baumgarte, J., 'Stabilization of constraints and integrals of motion in dynamical systems', Computer Methods in Applied Mechanics and Engineering, 1, 1972, 1-16.

67. Neto, M.A., Ambrósio, J., 'Stabilization methods for the integration of differential-algebraic equations in the presence of redundant constraints', Multibody Systems Dynamics, 10(1), 2003, 81-105.

68. Gear, C.W., 'Numerical solution of differential-algebraic equations', IEEE Transactions on Circuit Theory, CT-18, 1982, 89-95.

69. Shampine, L., Gordon, M., Computer solution of ordinary differential equations: the initial value problem, Freeman, San Francisco, California, 1975.

70. IMSL Math Library, Visual Numerics Inc., Huston, Texas, 1997.

71. Matlab, Mathworks Inc., Natick, Massachusetts, 2008. 